\title{
Risk prediction models for contrast induced nephropathy: systematic review
}

The name of the second author listed in this Research paper (BMJ 2015;351:h4395, doi:10.1136/bmj.h4395) should be Prakesh S Shah (not Prakesh M Shah).
Cite this as: BMJ 2015;351:h5401

๑ BMJ Publishing Group Ltd 2015 